\section{Background}

Motor theories: speech sounds perceived directly as articulatory gestures $^{1}$

- Sensorimotor theories: speech sounds perceived as sounds; auditory targets delimit production through corrective motor feedback. Motor information used if perception is hindered ${ }^{2,3}$

- L2: Perception \& production accuracy in L2 not always correlated ${ }^{4}$ : high individual variability

\section{Method ${ }^{5}$}

- 17 Spanish-dominant bilinguals \& 9 controls produced English vowel pairs /æ/,/e/ and Spanish /a/,/e/

- Individual categorical perception test stimuli resynthesised from each speaker's vowels, using 8 black dot coordinates to generate F1, F2 (see below)

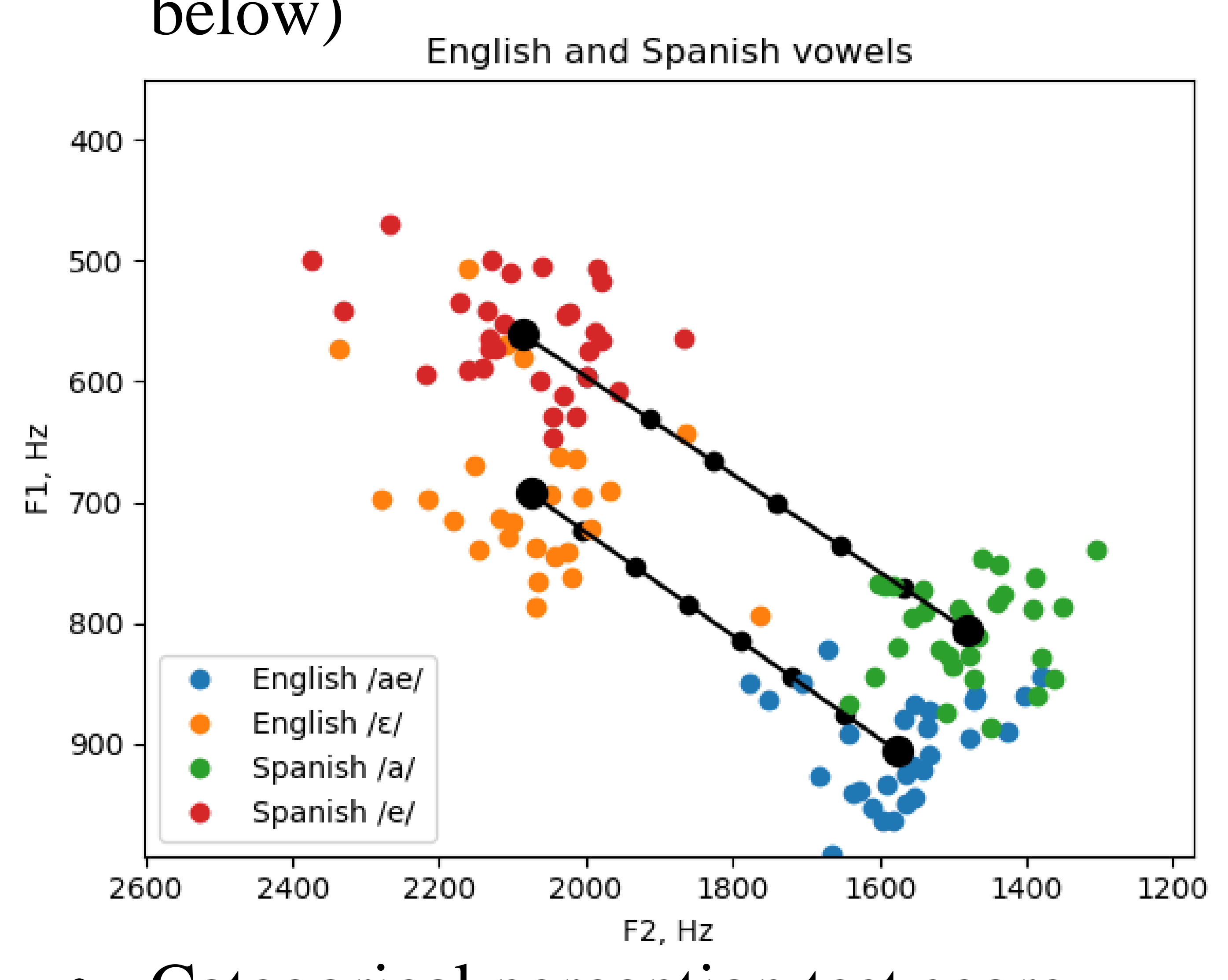

- Categorical perception test score multiplied by vowel mean distance/8 to calculate perceptual boundary

\title{
What is the relationship between speech perception and production in bilinguals?
}

Maddy Rees, Dept. of Theoretical \& Applied Linguistics, University of Cambridge mljr2@cam.ac.uk

\section{Results}

- VBB (Vowel Variability Based Boundary) x CPB (Categorical Perceptual Boundary) correlated significantly in bilinguals speaking (L2) English, and monolingual controls

- Correlation not significant in bilinguals speaking Spanish

- Speakers bootstrapping vowel boundaries in less-differentiated language, potentially from access to subvocal rehearsal/phonological loop during perception ${ }^{6}$

- Low correlation between production/ perception suggests primarily auditory processing ${ }^{7}$ in bilinguals speaking Spanish

- Fast inter-stimulus intervals hinder access to phonological loop ${ }^{8}:$ Spanish speakers may process stimuli/perform task faster

- Less crowded vowel space in Spanish, so no additional motor information needed for perceptual differentiation

\section{Conclusions}

- Result supports sensorimotor over motor models: if perception were entirely gesture-based, CPB and VBB would be mapped to the same location in vowel space regardless of processing speed/vowel space crowding

- Integration of auditory and motor information a useful processing strategy, especially when vowel spread higher or perceptual information unclear

- Further research: effects of ambient language, L2 proficiency and processing speed on correlation, adding in Spanish monolinguals

\section{References \& Acknowledgements}

1.Liberman, A.M.., \& Mattingly, I.G. (1989). A specialization for speech perception. 2. Hickok, G., \& Poeppel, D. (2007). The cortical organization of speech 3. Guenther, F. H. \& V Vladusich, T. (2012). A neural thent production. Journal of Neurolinguistics, 25(5), 408-422. 4. Sakai, M., \& Mooman, C. (2018). Can perception training improve the production training research.Applied Psycholinguistics, 39(1), 187-224. 5. Chao, S. C., Ochoa, D., \& Daliri, A. (2019). Production variability and categorical perception of owele are strongly linked. Frontiers in Human Neuroscience, $13,1-7$
6. Baddeley, A., Lewis, V., \& Vallar, G. (1984). Exploring the articulatory loop. The Quarterly Journal of Experimental Psychology Section A, 36(2), 233-252. 7. Callan, D. E., Jones, J. A., Callan, A. M., \& Akahane-Yamada, R. (2004). Phonetic
perceptual identification by native-and second-language speakers differentially perceptual identification by native-and second-language speakes
activates brain regions involved with acoustic phonetic processing and those involved with articulatory-auditiory/orosensory internal models. Neurolmage, 22(3), 1182 8. Peperkamp, S., and Bouchon, C. (2011). The relation between perception and Floderence: ISCA.

I wish to thank ESRC DTP \& Pembroke College for funding my Masters year, as well my supervisor and participants.
Cambridge ESRC Doctoral Training Partnership 